\title{
Caractéristiques des feux dans un incendie en savane soudanienne au Tchad
}

\author{
Madjimbe GUIGUINDIBAYE $^{1 *}$, Mamounata O. BELEM ${ }^{2}$ et Joseph I. BOUSSIM ${ }^{1}$ \\ ${ }^{1}$ Université de Ouagadougou. Unité de Formation et de Recherche en Sciences de la vie et de la Terre. \\ Laboratoire de Biologie et Ecologie Végétales, 03 BP : 7021 Ouagadougou 03, Burkina Faso. \\ ${ }^{2}$ Centre National de la Recherche Scientifique et Technologique. Institut de l'Environnement et de Recherches \\ Agricole. Département Productions Forestières. 03 BP : 7047 Ouagadougou 03, Burkina Faso. \\ *Auteur correspondant, E-mail : madjimbe1@yahoo.fr; Tél : +23566393529.
}

\section{RESUME}

La présente étude a été entreprise dans la forêt de Guirkouh en zone soudanienne dans le but de contribuer à la connaissance des caractéristiques des feux expérimentaux dans un incendie au Tchad. Le comportement d'un feu de végétation peut être caractérisé de diverses manières : la température du feu atteinte à divers niveaux, la vitesse de propagation, la hauteur des flammes, la superficie couverte par le feu et l'intensité du feu. Une étude de la biomasse maximale de la strate herbacée a été estimée à 2,107 t MS/ha par la méthode de la récolte intégrale. L'étude des caractéristiques des feux a révélé que les températures sont différentes selon l'emplacement des capteurs (dans le sol, au niveau de sol et dans la végétation). La température maximale obtenue est de $593{ }^{\circ} \mathrm{C}$ à hauteur de $50 \mathrm{~cm}$. En moyenne une valeur de 4,75 m pour la hauteur des flammes, 90\% comme proportion de surface couverte par le feu. L'intensité du feu atteint 594,77 $\mathrm{KJ} / \mathrm{s} / \mathrm{m}$. La vitesse de propagation du feu dépend étroitement de la vitesse du vent et de la hauteur de la végétation. La hauteur de la flamme quant à elle est fonction de la vitesse de la propagation du feu et de la hauteur de la végétation. La vitesse et le sens du vent se sont révélés influencer significativement le comportement du feu. Les résultats obtenus permettent de mieux comprendre le comportement du feu dans les savanes soudaniennes. Ils présentent aussi une grande importance pour les opérations de lutte anti-incendie. (C) 2013 International Formulae Group. All rights reserved.

Mots clés : Comportement des feux, Tchad, Forêt de Guirkouh, Zone soudanienne, lutte anti-incendie.

\section{INTRODUCTION}

Les feux de brousse incontrôlés sont reconnus comme source de dégradation des ressources forestières et d'autres ressources naturelles terrestres. Ils ont un impact négatif évident sur la dynamique des phytocénoses par leur rôle majeur dans la dégradation et les contraintes de la régénération naturelle (Sonko, 2000 ; Ouedraogo, 2006 ; Jacquin, 2010). Les feux de brousse maintiennent chaque formation dans son état, empêchant la savane d'évoluer en forêt, et ne permettant pas aussi à la forêt d'envahir la savane, les espèces végétales régénérant après les feux (Sawadogo et Fournier, 2004). Ils constituent un fléau dans les pays du sahel dont le Tchad. $\mathrm{Au}$ Tchad particulièrement, ils sont surtout violents dans le sud, le centre du pays, et le long de la frontière avec la République Centrafricaine. C'est dans ces zones qu'on observe beaucoup d'éleveurs transhumants tchadiens en période sèche à la recherche de 
pâturage et de points d'eau. Selon la Statistique de la Direction Générale des Forêts, chaque année, plus de 37500 ha de végétation sont brûlés par les incendies.

Pour connaître le « comportement du feu », nous avons effectué une série de brûlages sur des placettes situées dans la forêt de Guirkouh au sud du Tchad. Le choix de cette forêt s'explique par le fait qu'elle abrite des réserves naturelles, où les pratiques de feux sont courantes.

L'expression «comportement du feu » est utilisée pour désigner les divers phénomènes qui apparaissent au cours d'un feu (Mbow, 2000). Le comportement des feux est sous l'influence de certains facteurs bien connus des auteurs travaillant sur les feux de brousse (Trollope et al., 2002; Biligili et Saglam, 2003).

Ces phénomènes sont si nombreux et si changeants qu'il est impossible de les cerner tous. Il s'agit de la biomasse herbacée combustible et les éléments des conditions météorologiques qui ont précédé la mise à feu: la température ambiante, l'humidité relative, l'humidité du sol, le vent. C'est à travers la mesure de certains paramètres qu'on évalue le comportement du feu. Nous avons retenu à cet effet, cinq (5) paramètres d'évaluation: la température du feu atteinte à divers niveaux $(-10 \mathrm{~cm} ;-5 \mathrm{~cm} ; 0$ $\mathrm{cm} ; 5 \mathrm{~cm} ; 10 \mathrm{~cm} ; 25 \mathrm{~cm} ; 50 \mathrm{~cm} ; 100 \mathrm{~cm}$ et $150 \mathrm{~cm}$ ), la vitesse de propagation, la hauteur des flammes, la superficie couverte par le feu et l'intensité du feu qui en sont de bons exemples selon la littérature.

L'objectif de ce travail est d'étudier les facteurs influençant le comportement du feu et les paramètres d'évaluation de ce comportement.

\section{MATERIEL ET METHODES}

\section{Présentation de la zone d'étude}

La forêt de «Guirkouh» qui signifie forêt dense ou buisson d'arbres en sara, langue de cette localité, site de notre recherche, est située au sud du Tchad. Elle est comprise entre les latitudes $8^{\circ} 55^{\prime} 17^{\prime \prime}$ et $9^{\circ} 00^{\prime} 05^{\prime \prime}$ Nord et les longitudes $18^{\circ} 19^{\prime} 44^{\prime \prime}$ et $18^{\circ} 25^{\prime 2} 24^{\prime \prime}$ Est (Figure 1). Il se localise dans le Département de Barkôh, canton Koumogo, à $25 \mathrm{~km}$ au sud de la ville de Sarh, chef lieu du Département. Sa superficie est de 6436 ha.

Le climat est de type SoudanoGuinéen. Il est caractérisé par l'alternance de deux saisons: une saison sèche (octobre à mars) et une saison pluvieuse (avril à septembre). La température moyenne est de $28{ }^{\circ} \mathrm{C}$. La moyenne des températures maximales est de $33{ }^{\circ} \mathrm{C}$ et celle des températures minimales est de $22{ }^{\circ} \mathrm{C}$. La pluviométrie moyenne annuelle de 1978 à 2010 est de $1100 \mathrm{~mm}$ à Sarh.

La zone est formée d'une plaine plate inondable dont l'altitude varie entre 326 et $388 \mathrm{~m}$. Le sol est ferrugineux tropical. La végétation de la forêt de Guirkouh est constituée de forêts claires, de savanes herbeuses et principalement de savanes arborées et arbustives dominées par Khaya senegalensis, Daniella oliveri, Prosopis africana, Vitellaria paradoxa, ainsi que des forêts claires à Anogeissus leiocarpus. Detarium microcarpum, Ximenea americana et Piliostigma thonningii sont également bien représentés dans cet écosystème. Les savanes sont prédominantes, ce qui explique la fréquence des feux de brousse dans cette zone.

Le fleuve barkôh qui traverse cette forêt sur une longueur de 10,82 km est bordé par une végétation ripicole et les vallées comportent des galeries forestières avec des espèces comme Mitragyna inermis et Diospyros mespiliformis. La strate herbacée est dominée par les genres Andropogon, Hyparrhenia et Loudetia.

\section{Les facteurs influençant le comportement des feux \\ La biomasse herbacée}

Les herbacées jouent un rôle capital dans la propagation du feu. Elles propagent rapidement la flamme quand elles sont sèches, et lorsqu'elles sont gorgées d'eau, elles ralentissent notablement la vitesse de propagation. Pour quantifier la biomasse herbacée, six (6) carrés de biomasse ont été fauchés sur la parcelle avant le feu. Cette 
mesure a été reprise après le passage du feu pour évaluer la biomasse consumée par le feu. La teneur en eau de la biomasse a été évaluée après séchage à l'air des échantillons prélevés avant les feux. La hauteur de la strate herbacée a été évaluée en notant la hauteur moyenne dans chaque carré de biomasse.

\section{La température ambiante du milieu}

Son effet immédiat sur le comportement du feu est d'influencer par la température du combustible (herbacé) et ainsi la quantité de la chaleur requise pour atteindre son point d'inflammation (Trollope et al., 2002). Elle a été mesurée avant chaque mise à feu à l'aide d'un thermomètre.

\section{L'humidité de l'air}

C'est la proportion de vapeur d'eau se trouvant dans l'air à une température donnée. Elle a été mesurée à l'aide d'un hygromètre portable.

\section{L'humidité du sol au moment de la mise à feu}

Des échantillons de sol ont été prélevés sur les parcelles avant les mises à feu. Ces échantillons ont d'abord été pesés au moment de leur prélèvement puis séchés à l'étuve à $105{ }^{\circ} \mathrm{C}$ pendant 24 heures pour la détermination de la teneur en eau du sol pendant la mise à feu.

\section{Le vent}

Des auteurs ont trouvé des corrélations entre la vitesse du vent et la vitesse de propagation du front de flamme (Paulo, 2001 ; Bilgili et Sanglam, 2003). Dans cette étude, la vitesse du vent a été enregistrée pendant le feu à l'aide d'un anémomètre portable et sa direction est donnée par un morceau d'étoffe accroché au sommet d'un piquet. Nous n'avons pas placé notre anémomètre à $10 \mathrm{~m}$ au dessus du sol conformément à la position des stations de météo, dans la mesure où le vent qui influence la propagation de notre feu expérimental, est sans doute celui qui se déplace juste au-dessus de la végétation et de la couche combustible, c'est-à-dire entre 1,50 et $2 \mathrm{~m}$ environ.

\section{Les paramètres de comportement des feux} Température du feu atteinte à divers niveaux

À défaut de thermocouple, appareil approprié permettant d'évaluer simultanément les températures et leurs variations au cours du temps, nous avons utilisé des substances chimiques. Elles sont formées d'une série de vernis. Chaque vernis est caractérisé par une couleur et un point de fusion précis correspondant à une température connue. Nous avons utilisé les vernis pour peindre des plaquettes de micas de $5 \mathrm{~cm} \mathrm{x} 10 \mathrm{~cm}$ et après séchage, le côté peint de la plaquette de micas est recouvert d'un morceau d'amiante de même surface afin de protéger les bandes de peinture contre les dégâts de manipulation ou les dépôts de produits de combustion. Le mica et l'amiante sont utilisés parce qu'ils présentent l'avantage d'être incombustibles, et le fait qu'ils soient de mauvais conducteurs leur empêche d'avoir une action secondaire sur la fusion des vernis, sans empêcher le libre passage de la chaleur. Une heure avant la mise en feu nous avons accroché quarante de ces plaquettes sur des piquets, à différentes hauteurs et vingt autres ont été enterrées dans le sol ou placées dans la litière. Nous avons choisi les niveaux $-10 \mathrm{~cm} ;-5 \mathrm{~cm} ; 0 \mathrm{~cm} ; 5$ $\mathrm{cm} ; 10 \mathrm{~cm} ; 25 \mathrm{~cm} ; 50 \mathrm{~cm} ; 100 \mathrm{~cm}$ et 150 $\mathrm{cm}$.

\section{La vitesse de propagation du feu}

La vitesse de propagation du feu a été estimée à partir du temps mis par le front de flamme pour parcourir une distance de 10 mètres dans le sens du vent et contre le sens du vent. Le feu est d'abord allumé sur un côté de la parcelle dans le sens contraire à la direction du vent. Après la mise à feu, un chronomètre a suivi le front des flammes, en notant le temps qu'il met pour parcourir la distance de 10 mètres. Une autre similaire est effectuée dans le sens de la direction du vent. Sur le site, 4 parcelles ont subi ces feux précoces à la date du 20 novembre 2011. 
M. GUIGUINDIBAYE et al. / Int. J. Biol. Chem. Sci. 7(3): 1147-1156, 2013

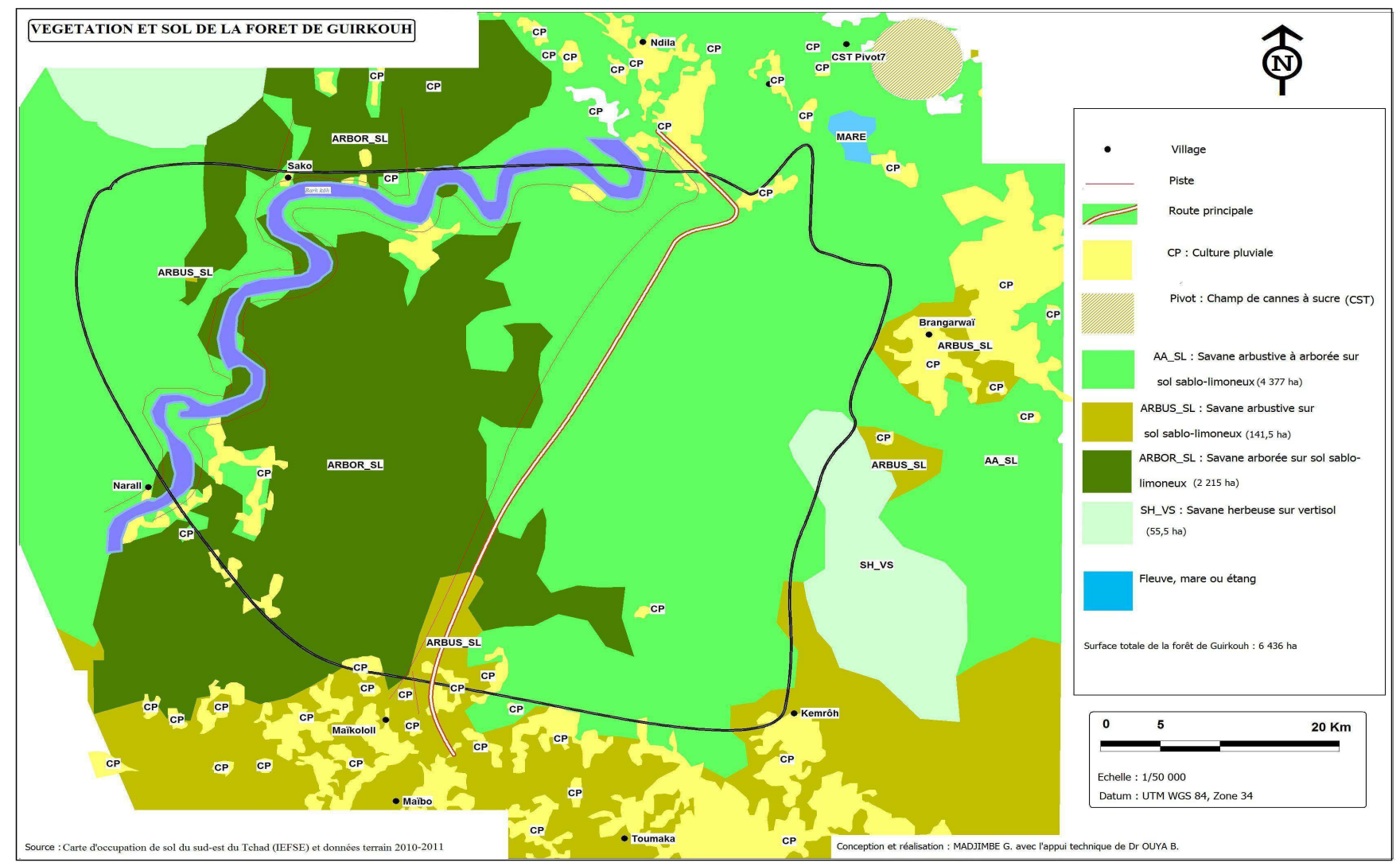

Figure 1 : Carte de végétation et de sol de la forêt de Guirkouh. 


\section{La hauteur des flammes}

Un feu peut brûler de plusieurs manières : on distingue les feux d'humus qui brûlent la matière organique issue de la saison passée en ne dégageant pas de flamme, les feux de surface qui brûlent la biomasse herbacée se trouvant sur place avec production de flamme n'atteignant pas les cimes des arbres et les feux de couronne atteignant les cimes des arbres. Toutes ces catégories de feux s'observent concomitamment sur le terrain. La hauteur des flammes a été mesurée de la façon suivante : le long d'un côté de la parcelle où est mis le feu, à un intervalle de 10 mètres, 5 perches de 6 mètres de long ont été installées. La hauteur des flammes est obtenue en faisant la moyenne des hauteurs enregistrées au niveau de chaque perche.

\section{La superficie couverte par le feu}

Elle a été estimée après le passage du feu sur les parcelles. La superficie couverte est obtenue en notant la proportion de superficie brûlée dans les 25 placettes que compte chaque parcelle. Les codifications retenues à cet effet sont celles de Dayamba (2005):

$0=$ la placette n'est pas atteinte par le feu

$1=1 / 4$ de la placette est atteinte par le feu

$2=1 / 2$ de la placette est atteinte par le feu

$3=3 / 4$ de la placette est atteinte par le feu

$4=$ toute la placette est atteinte par le feu

\section{L'intensité du feu}

Elle se réfère à la chaleur libérée par unité de temps et par unité de distance parcourue par le front de flamme. Elle se calcule par la formule de Byram (1959): I = H.W.R où I est l'intensité du feu en $\mathrm{kj} / \mathrm{s} / \mathrm{m}$; W est la biomasse consumée en $\mathrm{kg} / \mathrm{m} 2$; R est la vitesse de propagation du front de flamme en $\mathrm{m} / \mathrm{s}$. H est une constante donnant le rendement calorifique de la biomasse en $\mathrm{kj} / \mathrm{kg}$.
Une valeur de 19000 a été utilisée basée sur les données de littérature (Bilgili et Saglam, 2003).

Pour analyser la liaison entre les différents paramètres au cours des feux, nous avons utilisé comme méthode la matrice des coefficients de corrélation de Pearson. La matrice est évidemment symétrique et sa diagonale est constituée de 1 puisque la corrélation d'une variable avec elle-même est parfaite. Une matrice de corrélation permet de détecter rapidement certaines liaisons, de tester si la liaison est statistiquement significative et de quantifier l'intensité de la liaison linéaire entre deux paramètres. Le coefficient de corrélation sert également à caractériser une relation linéaire positive ou négative. Plus il est proche de 1 (en valeur absolue), plus la relation est forte. Toutefois, dès que le nombre de paramètres devient important, les interprétations deviennent difficiles et on se tourne vers les analyses factorielles.

\section{RESULTATS}

\section{Les facteurs influençant le comportement des feux}

Les Tableaux 1 et 2 résument les caractéristiques de la biomasse au cours du feu expérimental et les paramètres climatiques (voir à la fin du manuscrit).

\section{Les paramètres de comportement des feux}

L'évaluation de la température du feu atteinte à divers niveaux, après dépouillement des plaquettes de vernis est reportée sous forme d'histogramme à la Figure 2.

Les données concernant la biomasse consumée, la vitesse de feu, la hauteur de flamme et l'intensité du feu sont résumés dans le Tableau 3, et les corrélations trouvées entre les différents paramètres au cours du feu dans le Tableau 4. 


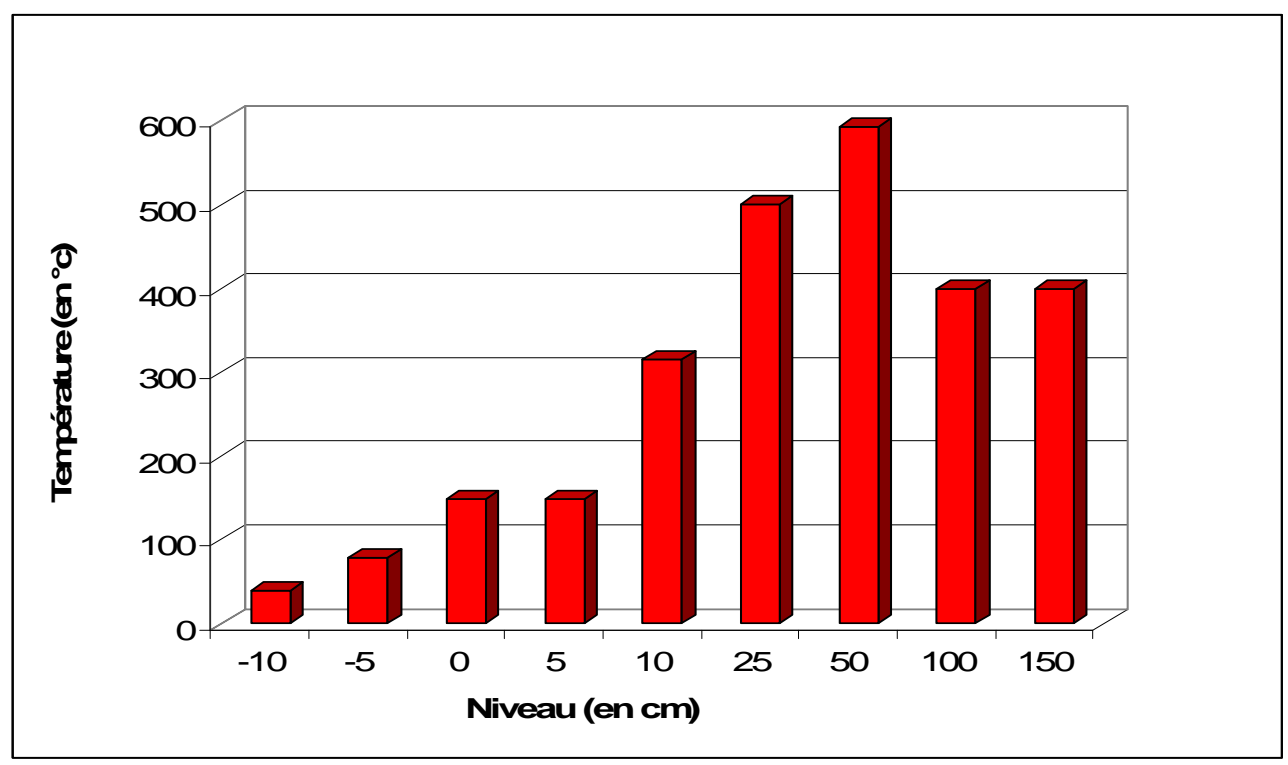

Figure 2 : Répartition des températures dans le sol et dans la végétation.

Tableau 1 : Caractéristiques de la biomasse au cours du feu expérimental.

\begin{tabular}{lcccc}
\hline Parcelles & $\begin{array}{c}\text { Heures de mise } \\
\text { du feu }\end{array}$ & $\begin{array}{c}\text { Hauteurs moyennes } \\
\text { de la biomasse (cm) }\end{array}$ & $\begin{array}{c}\text { Biomasse } \\
\text { Combustible }\left(\mathbf{g} / \mathbf{m}^{\mathbf{2}}\right)\end{array}$ & $\begin{array}{c}\text { Taux d'humidité } \\
(\mathbf{\%})\end{array}$ \\
\hline 1 & $6 \mathrm{~h} 25 \mathrm{mn}$ & $126 \pm 24$ & $189 \pm 164$ & 46,40 \\
2 & $7 \mathrm{~h} 20 \mathrm{mn}$ & $148 \pm 21$ & $283 \pm 260$ & 37,40 \\
3 & $8 \mathrm{~h} 10 \mathrm{mn}$ & $132 \pm 28$ & $334 \pm 254$ & 44,50 \\
4 & $8 \mathrm{~h} 48 \mathrm{mn}$ & $136 \pm 37$ & $321 \pm 152$ & 40,46 \\
\hline
\end{tabular}

Tableau 2: Les paramètres climatiques qui ont précédé la mise à feu.

\begin{tabular}{lllcc}
\hline Parcelles & DV & $\begin{array}{l}\mathbf{W} \\
(\mathbf{m} / \mathbf{s})\end{array}$ & $\begin{array}{c}\mathbf{T}^{\mathbf{0}} \\
\left.\mathbf{(}^{\mathbf{0}} \mathbf{C}\right)\end{array}$ & $\begin{array}{c}\text { HR } \\
(\mathbf{\%})\end{array}$ \\
\hline 1 & W-NW & 1,8 & 16,8 & 52 \\
2 & NE & 2,3 & 18,4 & 48 \\
3 & NE & 2,8 & 32,6 & 25 \\
4 & NW & 3,1 & 33,8 & 23
\end{tabular}

DV (Direction du vent); W (Vitesse maximale du vent au cours du feu en $\mathrm{m} / \mathrm{s}$ ); $\mathrm{T}^{\mathrm{o}}$ (Température ambiante du milieu en ${ }^{\circ} \mathrm{C}$ ) ; HR (Humidité relative de l'air en $\%$. 
M. GUIGUINDIBAYE et al. / Int. J. Biol. Chem. Sci. 7(3): 1147-1156, 2013

Tableau 3 : Les paramètres du comportement des feux.

\begin{tabular}{|c|c|c|c|c|c|c|}
\hline \multirow[t]{2}{*}{ Parcelles } & \multirow[t]{2}{*}{$\begin{array}{c}\text { FC } \\
\left(\mathrm{g} / \mathrm{m}^{2}\right)\end{array}$} & \multicolumn{2}{|c|}{$\begin{array}{c}\text { Vitesse du feu } \\
(\mathrm{m} / \mathrm{s})\end{array}$} & \multirow[t]{2}{*}{$\begin{array}{l}\mathbf{H F} \\
(\mathbf{m})\end{array}$} & \multirow[t]{2}{*}{$\begin{array}{l}\text { IFDV } \\
(\mathrm{KJ} / \mathrm{s} / \mathrm{m})\end{array}$} & \multirow[t]{2}{*}{$\begin{array}{l}\mathrm{IFCV} \\
(\mathrm{KJ} / \mathbf{s} / \mathbf{m})\end{array}$} \\
\hline & & DV & $\mathrm{CV}$ & & & \\
\hline 1 & 178 & 0,017 & 0,015 & 3,5 & 57,49 & 50,73 \\
\hline 2 & 259 & 0,033 & 0,016 & 5 & 162,39 & 78,73 \\
\hline 3 & 298 & 0,045 & 0,016 & 5 & 254,79 & 90,59 \\
\hline 4 & 301 & 0,104 & 0,018 & 5,5 & 594,77 & 102,94 \\
\hline
\end{tabular}

Tableau 4: Matrice de corrélation entre les différents paramètres au cours des feux.

\begin{tabular}{|c|c|c|c|c|c|c|c|c|c|c|}
\hline & VFDV & VFCV & IFDV & IFCV & FC & To & HR & HS & HF & $\mathbf{W}$ \\
\hline VFDV & 1,0000 & & & & & & & & & \\
\hline VFCV & $0,9862 * *$ & 1,0000 & & & & & & & & \\
\hline IFDV & 0,9988* & $0,9845 * *$ & 1,0000 & & & & & & & \\
\hline IFCV & 0,8557 & 0,8831 & 0,8764 & 1,0000 & & & & & & \\
\hline FC & $-0,0153$ & 0,1485 & $-0,0110$ & 0,2922 & 1,0000 & & & & & \\
\hline $\mathbf{T}^{\mathbf{o}}$ & 0,7970 & 0,7440 & 0,8236 & 0,8745 & $-0,1767$ & 1,0000 & & & & \\
\hline HR & $-0,8006$ & $-0,7533$ & $-0,8276$ & $-0,8901 * * *$ & 0,1413 & $-0,9994 *$ & 1,0000 & & & \\
\hline HS & $-0,3532$ & $-0,5028$ & $-0,3460$ & $-0,4892$ & $-0,9044 * * *$ & $-0,0053$ & 0,0380 & 1,0000 & & \\
\hline HF & 0,7735 & 0,8412 & 0,7916 & $0,9678 * *$ & 0,5214 & 0,7278 & $-0,7509$ & $-0,6838$ & 1,0000 & \\
\hline $\mathbf{W}$ & 0,8823 & 0,8806 & $\mathbf{0 , 9 0 3 8} * * * *$ & $0,9847 * *$ & 0,1220 & $0,9428 * * *$ & $-0,9526 * *$ & $-0,3368$ & $0,9091 * * *$ & 1,0000 \\
\hline
\end{tabular}

$\mathrm{W}$ (Vitesse maximale du vent au cours du feu en $\mathrm{m} / \mathrm{s}$ ); HF (Hauteur des flammes en $\mathrm{m}$ ); HS (Humidité du sol en \%); HR (Humidité relative de l'air en \%); $\mathrm{T}^{\circ}$ (Température ambiante du milieu en ${ }^{\circ} \mathrm{C}$ ) ;

FC (Biomasse consumée par le feu en $\mathrm{kg} / \mathrm{m}^{2}$ ) ; IFCV (Intensité du feu contre le vent en $\mathrm{KJ} / \mathrm{s} / \mathrm{m}$ ) ; IFDV(Intensité du feu dans la direction du vent en KJ/s/m); VFCV (Vitesse du feu contre le vent en m/s); VFDV (Vitesse du feu dans la direction du vent en $\mathrm{m} / \mathrm{s}$ ); * Corrélations significatives au seuil de probabilité de $1 \%$ oo ; ** Corrélations significatives au seuil de probabilité de $5 \%$; *** Corrélations significatives au seuil de probabilité de $10 \%$. 


\section{DISCUSSION}

Selon la hauteur à laquelle sont placées les plaquettes les températures sont plus ou moins différentes. A la surface du sol et à 5 $\mathrm{cm}$ très proche du niveau du sol, la température est la même et égale à $149^{\circ} \mathrm{C}$. La température la plus élevée est de $593{ }^{\circ} \mathrm{C}$ observée à $50 \mathrm{~cm}$ au-dessus du sol. Ces valeurs sont voisines de celles observées par certains auteurs Dayamba (2005) et Bekdouche (2010). Dans le sol, les températures observées sont peu élevées et elles diminuent rapidement avec la profondeur. C'est ainsi que la température passe de $149{ }^{\circ} \mathrm{C}$ à $79^{\circ} \mathrm{C}$ entre 0 à $-5 \mathrm{~cm}$; elle descend jusqu' au $39^{\circ} \mathrm{C}$ entre $-5 \mathrm{~cm}$ et $-10 \mathrm{~cm}$. Cela confirme que l'effet de la chaleur dégagée par la combustion des végétaux est très peu ressenti en profondeur. La conclusion de certains travaux stipule que les températures à partir de $-10 \mathrm{~cm}$ sont assez bien amorties par le sol.

Jusque là, peu d'auteurs ont publié des travaux sur les vitesses de propagation des feux. Rappelons néanmoins ceux de Dayamba (2005) qui a trouvé des vitesses de propagation qui varient entre $1,4 \mathrm{~cm} / \mathrm{s}$ et 13,3 $\mathrm{cm} / \mathrm{s}$ selon la direction du vent et la teneur en eau des végétaux lors des feux des Graminées dans le parc W au Burkina. Ses résultats sont semblables aux nôtres où, apparemment, les hauteurs maximales des flammes sont atteintes pour des vitesses de vent de l'ordre de $10 \mathrm{~cm} / \mathrm{s}$. Les résultats obtenus représentent, peut être, des valeurs inférieures à celles qui auraient pu être observées dans de grands feux, mais cela peut être expliqué d'une part, par la nature des espèces dominantes et d'autre part, par le changement brusque de la direction du vent. Ainsi, la vitesse et le sens $\mathrm{du}$ vent se sont révélés influencer significativement le comportement du feu. La vitesse maximale atteinte par le vent au cours du feu est allée grandissante avec l'heure de mise en feu. Il en est de même pour la hauteur des flammes, la vitesse de propagation du front de flamme et partant de l'intensité du feu.

Les valeurs des superficies couvertes par le feu ont été élevées (en moyenne 90\%). Cette moyenne avoisine celle trouvée par Sawadogo et Fournier (2004), Dayamba (2005) pour le feu de décembre. On observe une corrélation positive très forte de l'ordre de $0,9988(\mathrm{P}<0,001)$ entre l'intensité du feu dans la direction du vent (IFDV) et la vitesse du feu dans le sens du vent (VFDV). A ce même seuil, la corrélation est négative et forte ($0,9994)$ entre l'humidité relative de l'air (HR) et la température ambiante du milieu $\left(\mathrm{T}^{0}\right)$.

Au seuil de probabilité de 5\%, des corrélations positives très fortes ont été trouvées entre VFCV et VFDV $(0,9862)$; IFDV et VFCV $(0,9845)$; HF et IFCV $(0,9678)$; W et IFCV $(0,9847)$ et $\mathrm{W}$ et HR $(0,9526)$.

Au seuil de probabilité de $10 \%$, il existe des corrélations positives entre $\mathrm{W}$ et $\mathrm{T}^{\circ}$ $(0,9428)$; W et $\mathrm{HF}(0,9091)$; $\mathrm{FC}$ et $\mathrm{HS}$ $(0,9044)$ et IFDV et $\mathrm{W}(0,9038)$. A ce même seuil, la corrélation est négative entre IFCV et HR $(-0,8901)$.

D'autres auteurs ont aussi trouvé des corrélations entre les différents paramètres climatiques et le comportement du feu. Paulo (2001) trouve que la vitesse du feu est significativement corrélée, d'une part à la vitesse du vent au seuil de probabilité de $1 \%$ et d'autre part, à la température et à l'humidité relative de l'air au seuil de probabilité de 5\%. Bilgili et Sanglam (2003) ont trouvé au seuil de probabilité de $1 \%$ des corrélations positives entre : la vitesse du feu et l'intensité du feu $(0,92)$; la vitesse du feu et la vitesse du vent $(0,84)$; l'intensité du feu et la biomasse consumée $(0,57)$; l'intensité du feu et la vitesse du vent $(0,64)$. A ce même seuil, ils trouvent une corrélation négative de $-0,89$ entre la température et l'humidité relative.

Nos résultats obtenus sont assez proches de ceux trouvés par Dayamba (2005) au Burkina Faso où les types de végétation et 
le climat sont plus ou moins semblables à ceux du Tchad. Il a obtenu une corrélation positive très forte de l'ordre de 0,999 au seuil de $1 \%$ entre l'intensité du feu dans le sens du vent et la vitesse du feu dans le sens du vent. A ce même seuil, la corrélation est négative et forte $(-0,999)$ entre la température ambiante et l'humidité de l'air. Au seuil de probabilité de $5 \%$, il a trouvé une corrélation positive très forte de l'ordre de 0,948 entre intensité du feu dans la direction du vent et l'intensité du feu contre le sens du vent. A ce même seuil, une corrélation très forte $(0,968)$ existe entre l'intensité du feu contre le vent et la vitesse maximale du vent au cours du feu.

Les caractéristiques données dans les tableaux traduisent une légère sévérité du feu en cette période (fin novembre). Cela est dû probablement au fait que la forêt de Guirkouh est dominée par les herbacées annuelles (Louditia annua), carburant pour les feux et qui dessèchent plus vite que les herbacées vivaces.

\section{Conclusion}

Cette étude sur le «comportement du feu » dans la forêt de Guirkouh apporte des précisions sur le phénomène des feux de végétation dans la savane soudanienne. Quoique réalisée sur des parcelles expérimentales, les données présentées correspondent bien au comportement des feux sauvages dans ce type de végétation.

En effet, elles sont semblables aux résultats obtenus par d'autres chercheurs. Ce qui permet de supposer une grande convergence du comportement du feu quel que soit le peuplement végétal qui brûle.

Par ailleurs, l'application à la lutte antiincendie de tels résultats peut être importante. En effet, l'ensemble des informations fournies caractérise le comportement du feu compte tenu de la structure du peuplement végétal et des conditions météorologiques dominantes au moment du feu. Ceci devrait permettre aux gestionnaires d'espaces naturels forestiers de connaître très rapidement la situation d'un incendie dans ce type de peuplement; et donc de décider très rapidement du choix des moyens à mettre en œuvre pour lutter et maîtriser plus efficacement et plus rapidement le feu.

\section{REMERCIEMENTS}

Nous tenons à remercier sincèrement le Gouvernement Français qui par le projet SNRA-ARS2T au Tchad, nous a fourni les moyens financiers pour la collecte des données. Nous remercions également les personnes qui ont participé de loin ou de près à la réalisation de ce travail.

\section{REFERENCES}

Bekdouche F. 2010. Evolution après feu de l'écosystème subéraie de Kabylie (Nord Algerien). Thèse de Doctorat d'Etat en sciences agronomiques, option : Ecologie forestière. Université Mouloud MAMMERI de Tizi-Ouzou, Algérie, $175 \mathrm{p}$.

Bilgili E, Saglam B. 2003. Fire behaviour in maquis fuels in Turkey. Forest Ecology and Management, 184: 201-207.

Byram GM. 1959. Combustion of Forest Fuels, in Forest Fire: Control and Use Davis KP (ed). Mc Graw - Hill Book $\mathrm{C}^{\circ}$ : New York; 61- 81.

Dayamba SD. 2005. Influence des feux de brousse sur la dynamique de la végétation dans le Parc W-Burkina. Mémoire de fin d'études d'Ingénieur du Développement Rural : option élevage. IDR/UPB-Burkina Faso, p 27-28

Jacquin A. 2010. Dynamique de la végétation des savanes en lien avec l'usage des feux à Madagascar. Thèse de Doctorat de l'Université de Toulouse, p. 15-16.

Mbow C. 2000. Etude des caractéristiques spatio-temporelles des feux de brousse et de leurs relations avec la végétation dans le parc Niokolo Koba (Sud-Est du 
sénégal). Doctorat de troisième cycle. Université de Dakar, p. 56-62.

Ouedraogo A. 2006. Diversité et dynamique de la vegetation ligneuse de la partie orientale du Burkina Faso. Thèse de Doctorat. Université de Ouagadougou, Burkina Faso, 195 p.

Paulo AMF. 2001. Fire spread prediction in shrub fuels in Portugal. Forest Ecology and Management, 144: 67-74.

Sawadogo L, Fournier A. 2004 - Influence de différents régimes de feu sur la dynamique de la végétation du Parc W. Rapport de consultation, 29 p.
Sonko I. 2000. Etude des effets de différents régimes de feux sur la flore et la végétation ligneuse des plateaux du Parc National du Niokolo (sud-est du Sénégal). Thèse de Doctorat de troisième cycle en Sciences de l'Environnement. Université Cheikh Anta Diop, Dakar, 125 p.

Trollope WS, Troloppe LA, Hartnett OC. 2002. Fire Behaviour a Key Factor in the Ecology of African Grasslands and Savannas, Viegas (ed). Forest Fire Research \& Wildland Fire Safety, Millpress: Rotterdam; 17 p. 